\title{
AGN lifetimes in UV-selected galaxies: a clue to supermassive black hole-host galaxy coevolution
}

\author{
Xiaozhi Lin ${ }^{1,2}$, Yongquan Xue ${ }^{1,2}$, Guanwen Fang ${ }^{3}$, Lulu Fan ${ }^{1,2,4}$, Huynh Anh N. Le ${ }^{1,2,5}$, and \\ Ashraf Ayubinia ${ }^{1,2}$ \\ 1 CAS Key Laboratory for Research in Galaxies and Cosmology, Department of Astronomy, \\ University of Science and Technology of China, Hefei 230026, China ; xzlin@ mail.ustc.edu.cn, \\ xuey@ustc.edu.cn \\ 2 School of Astronomy and Space Science, University of Science and Technology of China, Hefei \\ 230026, China \\ 3 School of Mathematics and Physics, Anqing Normal University, Anqing 246133, China \\ 4 Shandong Provincial Key Lab of Optical Astronomy and Solar-Terrestrial Environment, Institute of \\ Space Science, Shandong University, Weihai, 264209, China \\ ${ }^{5}$ CAS President's International Fellowship Initiative (PIFI) Fellow
}

Received 20XX Month Day; accepted 20XX Month Day

\begin{abstract}
The coevolution between supermassive black holes (SMBHs) and their host galaxies has been proposed for more than a decade, albeit with little direct evidence about black hole accretion activities regulating galaxy star formation at $z>1$. In this paper, we study the lifetimes of X-ray active galactic nuclei (AGNs) in $U V$-selected red sequence (RS), blue cloud (BC) and green valley (GV) galaxies, finding that AGN accretion activities are most prominent in $\mathrm{GV}$ galaxies at $z \sim 1.5-2$, compared with $\mathrm{RS}$ and $\mathrm{BC}$ galaxies. We also compare AGN accretion timescales with typical color transition timescales of $U V$-selected galaxies. We find that the lifetime of GV galaxies at $z \sim 1.5-2$ is very close to the typical timescale when the AGNs residing in them stay in the high-accretionrate mode at these redshifts; for BC galaxies, the consistency between the color transition timescale and the black hole strong accretion lifetime is more likely to happen at lower redshifts $(z<1)$. Our results support the scenario where AGN accretion activities govern $U V$ color transitions of host galaxies, making galaxies and their central SMBHs coevolve with each other.
\end{abstract}

Key words: galaxies: high-redshift — galaxies: evolution — galaxies: formation galaxies: supermassive black holes

\section{INTRODUCTION}

The coevolution of supermassive black holes (SMBHs) and their host galaxies has been one of the most fundamental questions in galaxy physics in the past two decades, since the discovery of the tight relation between black hole mass $\left(M_{\mathrm{BH}}\right)$ and stellar velocity dispersion $(\sigma)$, i.e., $M_{\mathrm{BH}}-\sigma$ relation (e.g., Ferrarese \& Merritt 2000; Gebhardt et al. 2000; Kormendy et al. 2011; Kormendy \& Ho 2013; Saglia et al. 2016). Over three orders of magnitude, the correlation between black hole mass and stellar velocity dispersion only has an scatter of $\sim 0.3$ dex. The studies of luminosity functions (and/or stellar mass functions) have also revealed that the peaks of the global galaxy star formation and black hole accretion 
are both at $z \sim 1.5-2$, and both show "downsizing" features, i.e., more massive galaxies/black holes started to exist and grow at earlier epoches of the cosmic time (e.g., Miyaji et al. 2000; Hopkins et al. 2007; Croom et al. 2009). Such evidence has shown that SMBHs and their host galaxies are very likely to coevolve with each other. In this case, some internal feedback mechanisms may be at work to govern the evolution.

One of the most popular scenarios to explain the coevolution between SMBHs and their host galaxies is through active galactic nucleus (AGN) feedback. The galaxy star formation and the central black hole accretion are fueled by the gas reservoirs within the same galaxy. When the dark matter halo grows to some critical values (e.g., Hickox et al. 2009; Lin et al. 2019, 2021), gas-rich mergers or disk instabilities (e.g., Martig et al. 2009; Leauthaud et al. 2012; Zolotov et al. 2015) may cause the gas clumps to lose their angular momenta, fall down to the galaxy center, and enhance the star formation activities, which would further trigger the AGN feedback activities, depleting the surrounding gas, suppressing the galaxy star formation, and making the stellar component and central SMBH grow synchronously (Merloni et al. 2010; Graham 2012; Kormendy \& Ho 2013; Sun et al. 2015; Saglia et al. 2016).

Nonetheless, there has been little direct observational evidence showing that AGN feedback would definitely quench galaxy star formation at high redshifts (Torres-Papaqui et al. 2020). The reasons, on one hand, lie in the observational difficulties in galactic scales at these redshifts. On the other hand, the timescales that AGNs stay active (in the Eddington or super-Eddington phase), usually being several tens of million years, are much shorter than the typical star formation timescales of galaxies (more than several hundred million years) (Bell 2002; Martini 2004; Adelberger et al. 2005; Hopkins et al. 2005; Hopkins \& Hernquist 2009). Thus, it is difficult to observe the interactions between AGN feedback and galaxy global star formation activities in the early universe.

In galaxy physics, the color bimodality (i.e., blue vs. red) of the galaxy distribution has long been known effective in classifying and studying the star-forming and quiescent galaxies up to high redshifts (Strateva et al. 2001; Blanton et al. 2003; Bell et al. 2004; Giallongo et al. 2005; Borch et al. 2006; Franzetti et al. 2007; Cirasuolo et al. 2007; Cassata et al. 2008; Whitaker et al. 2011; Lee et al. 2015). Although some dust-reddened star-forming galaxies may contaminate the red population, some researchers modified the separation criterion to improve the galaxy classification. For example, Brammer et al. (2009) introduced the dust-attenuation factor into the color separation criterion, making it more effective to classify the intrinsic star-forming and quiescent galaxies. Wang et al. (2017) checked out this new separation criterion, making it more self-consistent from the local to the high redshift universe $(z \sim 2.5)$.

There is a subtle special population of galaxies lying in the color conjunction region between the red and blue bimodality, which are the so-called green valley (GV) galaxies (e.g., Brammer et al. 2009; Xue et al. 2010; Wetzel et al. 2012; Fang et al. 2013; Schawinski et al. 2014; Chang et al. 2015). These galaxies usually show strong post-starburst features (e.g., Rowlands et al. 2018), and are considered as experiencing the quenching of star formation. Studying the relevant properties of these galaxies as well as their evolution with redshift hence can help to identify whether AGN feedback activity plays an important role during galaxy quenching. Some studies have revealed that the fraction of galaxies hosting AGNs is higher in GV galaxies, compared to red sequence (RS) and blue cloud (BC) galaxies (e.g., Silverman et al. 2008; Schawinski et al. 2010; Wang et al. 2017). However, most of the evidence about the AGN activities interacting with the star formation within these galaxies is indirect (e.g., Silk \& Rees 1998; Ishibashi \& Fabian 2012; Zubovas et al. 2013; Cresci et al. 2015; Le et al. 2017a,b; Shin et al. 2019). If AGN feedback indeed drives the quenching within these galaxies, the timescales that the central black holes remain active should be comparable to the color transition timescales of GV galaxies.

Lin et al. (2021) measured the lifetimes of massive GV galaxies at high redshifts based on the clustering analyses. The results can be rough estimates of the typical quenching timescales within these galaxies, shifting from the star-forming (BC) to quiescent (RS) phase, and are shown to be well consistent with the results from spectral energy distribution (SED) modeling (also see Lin et al. 2021). Meanwhile, measuring the lifetimes of AGNs within these galaxies is also possible. Hopkins \& Hernquist (2009) quantified the quasar lifetimes through their Eddington ratio $\left(\lambda_{\text {Edd }}\right)$ distribution. They demonstrated that, as long as the lifetimes of AGNs are short relative to the Hubble time, probing their 
lifetimes uniquely through the Eddington ratio distribution is applicable. Therefore, if we know the distributions of Eddington ratio in RS, BC and GV galaxies, we can estimate the corresponding lifetimes of AGNs among these galaxies, and the results can be directly compared to the typical color transitional timescales of galaxies (i.e., lifetimes of galaxies of specific colors). In this way, we can examine the roles that AGNs play in quenching galaxy star formation.

Fortunately, Wang et al. (2017) provided the Eddington ratio distributions of AGNs in the galaxies of different extinction-corrected $U V$ colors through a systematic statistics of the fractions of AGNs of different accretion rates. Based on their results, we can estimate the lifetimes of AGNs of different accretion rates in galaxies of different colors in each specific cosmic epoch. Thus, it is possible to compare the AGN lifetimes with the typical color transitional timescales of galaxies and check the current quenching scenario, pinning down whether AGN feedback is important, especially in GV galaxies.

The outline of this paper is as follows. In Section 2, we introduce the method we adopt to compute the lifetimes of AGNs in RS, BC and GV galaxies. In Section 3, we describe our adopted Eddington ratio distributions. In Section 4, we discuss our results and compare the lifetimes of AGNs with the typical color transitional timescales in the three galaxy populations. We summarize our results and conclusions in Section 5.

Throughout this paper, we adopt a flat cosmology with $\Omega_{\mathrm{M}}=0.3, \Omega_{\Lambda}=0.7, H_{0}=$ $70 \mathrm{~km} \mathrm{~s}^{-1} \mathrm{Mpc}^{-1}$, and a normalization of $\sigma_{8}=0.84$ for the matter power spectrum.

\section{METHOD TO COMPUTE THE LIFETIMES OF AGNS}

We compute the lifetimes of AGNs utilizing the method discussed in Hopkins \& Hernquist (2009). This method is based on the Eddington ratio distribution of AGNs.

In general, as long as the lifetimes of AGNs are short compared to the Hubble time at a given luminosity or Eddington ratio (i.e., cosmological evolution of triggering rates can be ignored in the redshift bin concerned), the duty cycle $\delta$ of a certain type of AGNs can be expressed not only by the fraction of active accretion time over the full time interval, but also by the fraction of number counts/density of such AGNs over the whole AGN population. In this circumstance, we can translate the distribution of duty cycle at different Eddington ratios into the lifetimes of AGNs (also see Equation 3 in Hopkins \& Hernquist 2009):

$$
\frac{d \delta}{d \log \lambda}=\frac{1}{t_{H}(z)} \frac{d t}{d \log \lambda} \quad\left(t \ll t_{H}\right),
$$

where $t_{H}(z)$ is the Hubble time at the given redshift, and $\lambda$ (or $\lambda_{\mathrm{Edd}}$ ) is the Eddington ratio defined as:

$$
\lambda \equiv \frac{L_{\mathrm{bol}}}{L_{\mathrm{Edd}}}=\frac{L_{\mathrm{bol}}}{1.3 \times 10^{38} \operatorname{erg~s}^{-1}\left(M_{\mathrm{BH}} / M_{\odot}\right)} .
$$

On the other hand, the duty cycle of AGNs can also be related to the black hole number density function $\Phi$ and Eddington ratio distribution $P(\lambda)$ at a given mass and accretion rate (also see Equation 4 in Hopkins \& Hernquist 2009):

$$
\frac{d \delta}{d \log \lambda}=\frac{d n\left(\lambda, M_{\mathrm{BH}}\right) / n\left(M_{\mathrm{BH}}\right)}{d \log \lambda}=\frac{\Phi\left(\lambda \mid M_{\mathrm{BH}}\right)}{n\left(M_{\mathrm{BH}}\right)}=\frac{\Phi\left(\lambda \mid M_{\mathrm{BH}}\right)}{\int_{-\infty}^{+\infty} \Phi\left(\lambda \mid M_{\mathrm{BH}}\right) d \log \lambda}=\frac{d P\left(\lambda \mid M_{\mathrm{BH}}\right)}{d \log \lambda}
$$

Hence, the lifetimes of AGNs can be estimated using the combination of the above two equations.

Specifically, inverting Equation 1, we obtain:

$$
\frac{d t\left(\lambda \mid M_{\mathrm{BH}}\right)}{d \log \lambda}=t_{H}(z) \frac{d \delta}{d \log \lambda}
$$

Integrating duty cycle $\delta$ above $\lambda$, we obtain $t(>\lambda) \equiv t_{H}(z) \delta(>\lambda)$. This lifetime is directly determined by the observed Eddington ratio distribution $P(\lambda)$ at any redshift $\mathrm{z}$, and is often referred to as the implied 
"AGN lifetime" in various observational statistics. In physics, this lifetime represents the time that AGN systems spend on staying above the accretion rate $\lambda$ at a given redshift.

If the accretion triggering rate is relatively constant over the redshift range for AGNs of a given black hole mass, and the lifetime is short relative to the respective Hubble time, then the estimation through Equation 1 and Equation 3 is applicable. Furthermore, the Eddington ratio distribution of the observed AGNs, independent of any other constraints, can be directly translated into their lifetime distribution. Even if the accretion triggering rate strongly evolves with redshift and/or their characteristic lifetime is long compared to the Hubble time (e.g., low-redshift massive black holes, where their main growths had happened in much earlier epochs), utilizing the Eddington ratio distribution at a given redshift to predict the lifetime distribution of AGNs is still applicable. But, in this case, the actual duty cycle of an AGN is equivalent to their characteristic lifetime convolved with the distribution of accretion activity over the redshift bin of interest. Even so, in practice, accounting for the accretion triggering distribution over the redshift bin when mapping the observed Eddington ratio distribution does not significantly alter the shape of the lifetime distribution. In principle, this procedure only results in deriving a more accurate effective duty cycle than simply multiplying by $\sim 1 / t_{H}$. More relevant detailed discussions can be referred to Yu \& Lu (2004) and Hopkins et al. (2005, 2006a,b). Thus, once an observed AGN luminosity function (of a given black hole mass) is well constrained at each luminosity and redshift bin of interest, the corresponding Eddington ratio distribution can be uniquely translated into the lifetime distribution.

This lifetime, reflecting the total timescale an AGN spends on some accretion rate levels, may show a variation in galaxies of different extinction-corrected $U V$ colors. Comparing the AGN lifetimes in galaxies of different colors can give us the information about the duration of accretion activities of various levels in each redshift bin, which can help to examine the most recent AGN feedback scenario. Besides, the lifetimes of AGNs in these galaxies of different colors can also serve as the contrasts of the typical color transitional timescales of the respective galaxy populations. If it is indeed AGN feedback activity that drives the star-formation quenching, some similarities between the two timescales should be well identified.

At very high redshifts, the formula computed through the Eddington ratio distribution may underpredict the real lifetime of an AGN. This is because the Hubble time $t_{H}$ around these redshifts is very short, and if we do not consider AGN triggering physics varying significantly with redshifts, the lifetime of an AGN here would become comparable to the Hubble time. Thus, the condition in Equation 1 that $t \ll t_{H}$ would no longer hold. Nevertheless, this slightly under-estimated lifetime would still provide a meaningful comparison. We would discuss this influence on the final results and conclusions later in Section 4.

In this study, we also compare the lifetimes of AGNs with the lifetimes (i.e., typical color transitional timescales) of galaxies of different extinction-corrected rest-frame $U V$ colors (see Section 4.2). These lifetimes of galaxies are also computed through their duty cycles. But unlike the lifetimes of AGNs, the lifetimes of galaxies are derived based on their clustering results (Lin et al. 2019). The simple algorithm is as follows. We assume that for each dark matter halo having a mass similar to the measured halo mass of one of the three galaxy populations, the galaxy residing in it would undergo the respective color phase on that galaxy population during its evolution over cosmic time. According to this assumption, if we observe a galaxy whose halo mass is similar to one of the three galaxy populations but the galaxy does not exhibit the respective color, it means that this galaxy has not yet evolved into the phase of this galaxy population or it has already passed this phase. Thus the lifetime of a galaxy population here can be a rough estimate of the time that this galaxy is alive in a respective phase of color. Considering the quenching scenario, this galaxy lifetime can be also referred to as the "typical color transitional timescales of galaxies". In Lin et al. (2021), we have demonstrated that these galaxy color transitional timescales are consistent with the SED modeling results assuming an exponentially declining star-formation history. For more details, we refer the readers to Lin et al. (2021). 


\section{ADOPTED EDDINGTON RATIO DISTRIBUTIONS}

In this paper, we estimate the lifetimes of AGNs based on the Eddington ratio distribution of black holes in RS, BC and GV galaxies in the combination of GOODS-North and GOODS-South fields provided by Wang et al. (2017). They separated both AGN hosts and non-AGN hosts into RS, BC and GV galaxies according to their extinction-corrected rest-frame $U V$ colors. The X-ray data of these AGNs have good exposures, which are comprised of the 2Ms main point-source catalog in the GOODS-North field (Alexander et al. 2003) and the 4Ms main point-source catalog in the GOODS-South field (Xue et al. 2011). Then, they evaluated the AGN Eddington ratio distributions in the three populations of galaxies based on the statistics of their AGN fractions. During their statistics, they corrected for the observational incompleteness utilizing the $V_{\max }$ method, adopting the detection limits derived from the sensitivity maps in the GOODS-North (Alexander et al. 2003) and GOODS-South fields (Xue et al. 2011). Their detailed procedure is as follows. In each specific bin of redshift and stellar mass, they computed the fraction of AGNs falling in a fixed narrow bin of $\lambda_{\text {Edd }}$ around the median as the observed Eddington ratio distribution, $P\left(\lambda_{\mathrm{Edd}} \mid M_{*}, z\right)$. Then, they performed a simple linear fit to each of the Eddington ratio distribution,

$$
P\left(\lambda_{\mathrm{Edd}}\right) d \log \lambda_{\mathrm{Edd}}=A\left(\frac{\lambda_{\mathrm{Edd}}}{\lambda_{\text {Edd }_{\mathrm{cut}}}}\right)^{-\alpha} d \log \lambda_{\mathrm{Edd}},
$$

where A denotes the amplitude and $\alpha$ denotes the slope. $\lambda_{\mathrm{Edd}_{\mathrm{cut}}}$ is an arbitrary scaling factor, which was adopted as the Eddington luminosity, i.e., $\lambda_{\mathrm{Edd}_{\mathrm{cut}}}=1$. This power-law form of fitting was also suggested and adopted in a number of similar relevant studies (e.g., Aird et al. 2012; Trump et al. 2015; Jones et al. 2016).

Figure 1 shows the Eddington ratio distributions of the full AGN samples at $z=1$ and $z=2$ (black lines with dark and light grey regions) provided by Wang et al. (2017) in comparison with the local relation presented by Kauffmann \& Heckman (2009) (purple dash-dotted line) and the semi-analytic model predictions in Hopkins \& Hernquist (2009) at $z=0$ and $z=1$ (yellow dashed lines). Observationally, Kauffmann \& Heckman (2009) measured the Eddington ratios of local AGNs based on the extinctioncorrected [OIII] emission line luminosity. We adopted their Eddington ratio distribution selected with $10^{7} M_{\odot}<M_{\mathrm{BH}}<10^{8} M_{\odot}$ as analogues in the local universe, which corresponds to the galaxy stellar mass at $10^{10} M_{\odot}<M_{*}<10^{11} M_{\odot}$. This stellar mass range is roughly identical to the galaxy samples in Wang et al. (2017) and Lin et al. (2021). As in Wang et al. (2017), we apply a $10 \%$ normalization correction to the Eddington ratio distribution in Kauffmann \& Heckman (2009), since AGN hosts only comprise $\sim 10 \%$ in the total amount of galaxies at the stellar mass of $M_{*} \sim 10^{10.5} M_{\odot}$ (Trump et al. 2015) and Kauffmann \& Heckman (2009) only presented the $\lambda_{\text {Edd }}$ distribution in AGN host galaxies. Apart from the result in Kauffmann \& Heckman (2009), Hopkins \& Hernquist (2009) predicted the Eddington ratio distributions in different bins of black hole mass at $z=0$ and $z=1$. Their results were based on the best-fit observational quasar luminosity function as a function of redshift (Hopkins et al. 2007), as well as the best-fit semi-analytic lifetime distributions as a function of black hole mass in the hydrodynamic simulations of galaxy mergers incorporating with black hole growth (Hopkins et al. 2006a). After integrating them over the redshifts of interest with $M_{\mathrm{BH}}$ fixed, they obtained the Eddington ratio distribution of quasars in each specific bin of $M_{\mathrm{BH}}$. Same as the result quoted in Kauffmann \& Heckman (2009), we adopt the $\lambda_{\text {Edd }}$ distribution of AGNs in Hopkins \& Hernquist (2009) with a $M_{\mathrm{BH}}$ cut of $10^{7} M_{\odot}<M_{\mathrm{BH}}<10^{8} M_{\odot}$.

We note that utilizing the [OIII] emission line flux as the indicator of the AGN accretion rate (Kauffmann \& Heckman 2009) may not always be reliable, especially in the radiative inefficient regime (e.g., $L / L_{\mathrm{Edd}}<0.01$ ), where the ionized outflows and the star formation of the host galaxies may also produce low levels of [OIII] line fluxes. However, these influences are only restricted to the [OIII] radiative inefficient cases, where the AGN samples also suffer some degrees of selection incompleteness. And most importantly, in this paper, the AGN subsamples in Kauffmann \& Heckman (2009) are only served as the analogues of high-redshift AGNs in the local universe. Therefore, we do not further 


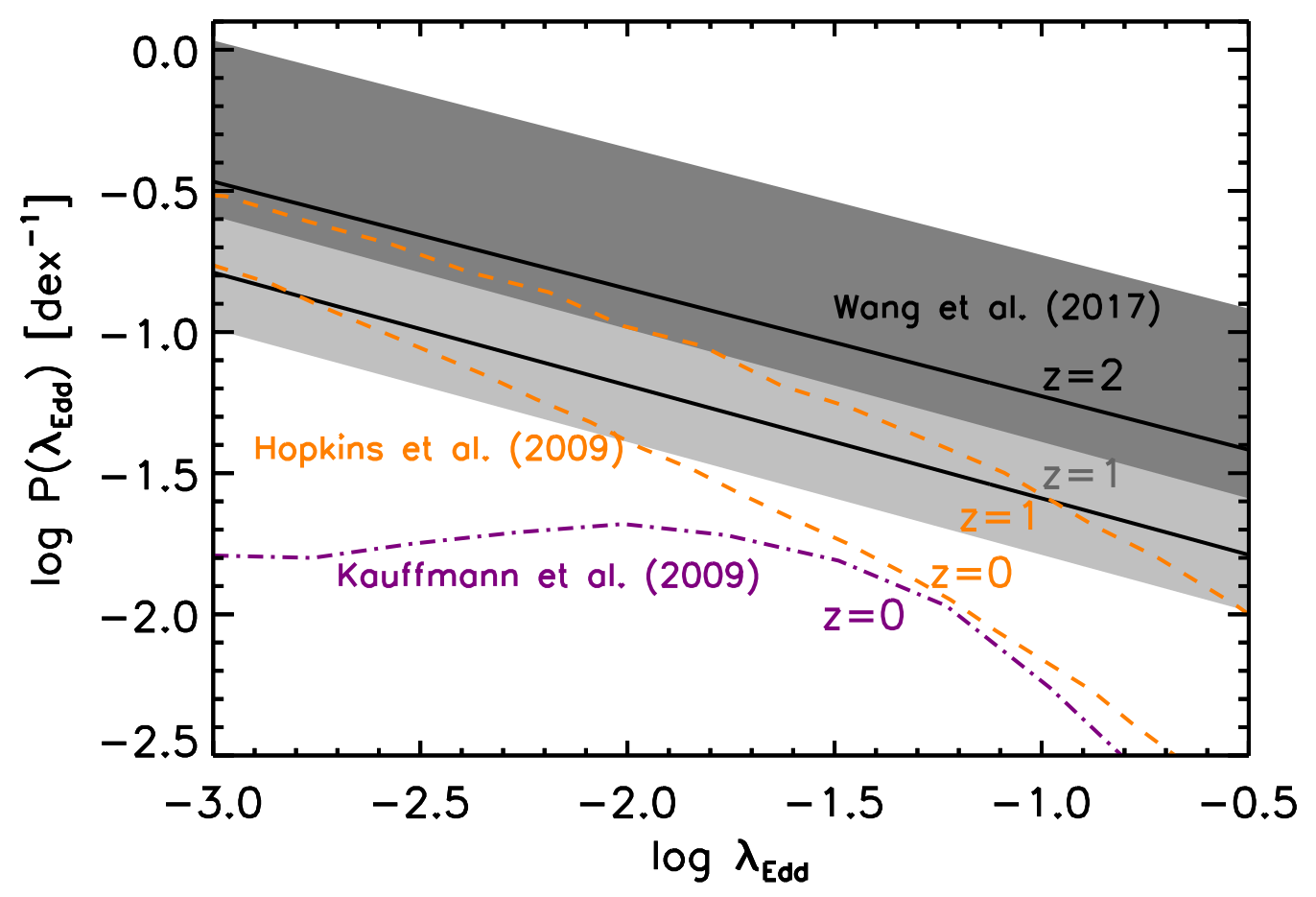

Fig. 1: Eddington ratio distributions of the full AGN samples at $z=1$ and $z=2$ (black lines with dark and light grey regions) provided by Wang et al. (2017) in comparison with the local relation presented by Kauffmann \& Heckman (2009) (purple dash-dotted line) and the semi-analytic model predictions in Hopkins \& Hernquist (2009) at $z=0$ and $z=1$ (yellow dashed lines). Both the observational local empirical relation presented by Kauffmann \& Heckman (2009) and semi-analytic model predictions in Hopkins \& Hernquist (2009) are restricted to the SMBH masses of $10^{7} M_{\odot}<M_{\mathrm{BH}}<10^{8} M_{\odot}$.

consider the deviation of estimating Eddington ratio from [OIII] emission line luminosity on the low accretion rate side.

In general, the Eddington ratio distributions of AGNs provided by Wang et al. (2017) show a good consistency when compared to the observational statistical relations and/or semi-analytic model predictions at different redshifts. Therefore, we do not consider any significant difference in the $\lambda_{\text {Edd }}$ distribution arising due to the observational field variance in this paper.

\section{RESULTS AND DISCUSSIONS}

In this section, we present and discuss the main results of this paper. In Section 4.1, we compare the evolution of Eddington ratio distributions of AGNs as a function of redshift among galaxies of different extinction-corrected rest-frame $U V$ colors. We also try to explain the possible physical mechanisms that are responsible for these trends. In Section 4.2, we display the AGN lifetimes among RS, GV and $\mathrm{BC}$ galaxies computed through the Eddington ratio distributions. We study their redshift evolutions and compare the results with the typical color transitional lifetimes in the three population of galaxies. In this way, we attempt to examine the possible roles of AGN accretion activities in the most current galaxy star-formation quenching scenario. 


\subsection{Eddington ratio distributions of AGNs among RS, GV and BC galaxies across different redshifts}

For each population of galaxies selected with different extinction-corrected rest-frame $U V$ colors, we compare the Eddington ratio distribution (Wang et al. 2017) with that of the local SDSS AGN host galaxies of different $4000 \AA$ break strength $\left(D_{n}(4000)\right)$ in Kauffmann \& Heckman (2009). In particular, the AGN host galaxies selected with $1.2<D_{n}(4000)<1.4,1.6<D_{n}(4000)<1.7$ and $1.7<D_{n}(4000)<1.9$ are chosen as the typical analogues of the BC, GV and RS galaxies in Wang et al. (2017), respectively. Considering that Wang et al. (2017) only provided the $\lambda_{\text {Edd }}$ distributions of galaxies at $z=1$ and $z=2$, we interpolate their best-fit amplitudes and slopes of the $\lambda_{\text {Edd }}$ distribution functions of RS, GV and BC galaxies, respectively, as a function of redshift to predict the Eddington ratio distribution of galaxies of each color at four more specific redshifts, i.e., $z=0.75, z=1.25$, $z=1.75$, and $z=2.25$. When accounting for the uncertainties of the Eddington ratio distribution, we keep the slope fixed for a clearer visualization. The result is shown in Figure 2.

It is clear that the Eddington ratio distributions of galaxies selected with different extinctioncorrected rest-frame $U V$ colors are closer to the local analogues of the respective $D_{n}(4000)$ indexes in Kauffmann \& Heckman (2009) towards lower redshifts. The redshift evolution of the distribution of $\lambda_{\text {Edd }}$ is much more significant in $U V$ redder galaxies. The AGN accretion rate in low-redshift or local RS galaxies is very low. These galaxies are usually considered as those having already consumed their gas supply, and both of their star formation and central black hole accretion tend to stop. While at higher redshifts, the difference between the $\lambda_{\text {Edd }}$ distributions of $U V$ red and blue galaxies becomes very small, especially around the medium and low accretion rates. This phenomenon may reflect the possible situation that gas supply to the central black hole has not yet been completely shut off in highredshift RS galaxies, thus their AGN accretion rates remain in a moderate level. On the other hand, the accretion rates of central black holes among $\mathrm{BC}$ galaxies do not show significant evolution with redshifts, which indicates that there may not be significant difference in the black hole accretion triggering mechanisms among these galaxies. BC galaxies are usually found to contain plenty of molecular gas supplies at whatever redshifts (e.g., Stark et al. 1986; Young et al. 1989; Sage et al. 1992; Taylor et al. 1999; Barone et al. 2000; Boselli et al. 2014; Cañameras et al. 2018; Tacconi et al. 2018). Hence, it is quite likely that the gas in these galaxies would freely assemble and fall down to feed the central black hole without any interruption.

There are a few possibilities that some dust-rich galaxies with moderate accretion rates would contaminate the RS galaxy population, especially at higher redshifts, i.e., $\mathrm{z}=1.75$ and $\mathrm{z}=2.25$. But since Wang et al. (2017) applied extinction-corrected rest-frame $U V$ colors to divide the galaxies, which is proven to be valid in separating the intrinsic red quiescent galaxies from the dust-reddened star-forming galaxies up to $z \sim 2.5$, we thus do not consider this contamination effect in this paper. It is also possible that some reverse mechanisms, such as star-formation rejuvenation, may compensate for the amount of galaxies lost during quenching. But since in most studies, galaxies discovered to be experiencing rejuvenation only make up a tiny fraction of the total amounts (Reipurth et al. 1997; Portegies Zwart 2000; Perets 2009), it is unlikely to significantly influence the global trends of galaxy quenching and the cosmic cooling. We thus also do not take this effect into account in this paper.

\subsection{AGN lifetimes in galaxies of different extinction-corrected rest-frame $U V$ colors across different redshifts}

Based on the Eddington ratio distributions of AGNs among each of the above three populations of galaxies, we compute their respective lifetimes through Equation 1 and Equation 3. The result is shown in Figure 3. In general, on the low accretion rate side, the lifetimes of AGNs among RS, BC and GV galaxies show no significant differences, especially at higher redshifts $(z>1)$. However, on the high accretion rate side, the lifetimes of AGNs in GV galaxies are obviously longer when compared to those of RS or BC galaxies, particularly at $z>1$. The trend is even clearer at $z>1.5$. These results indicate that strong AGN accretion is rather common in GV galaxies, in particular at higher redshifts $(z \sim 2)$. 


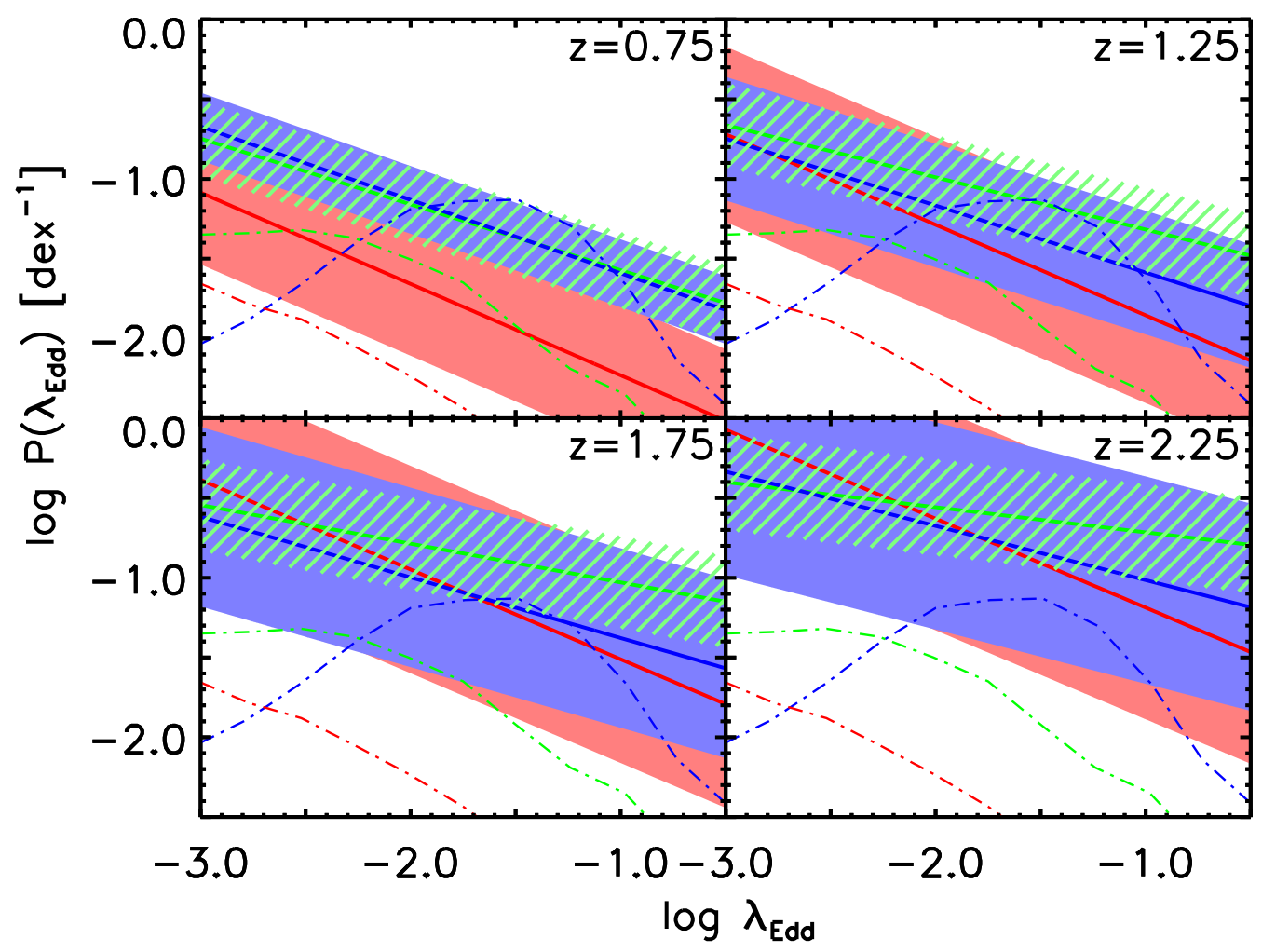

Fig. 2: Eddington ratio distributions in $\mathrm{BC}, \mathrm{GV}$ and RS galaxies at $z=0.75, z=1.25, z=1.75$, and $z=2.25$, shown as the thick solid color lines with the shaded/line-filled regions. The thin dashdotted lines in each panel are the same relations of the respective local AGN host analogues from Kauffmann \& Heckman (2009), selected with $1.2<D_{n}(4000)<1.4,1.6<D_{n}(4000)<1.7$ and $1.7<D_{n}(4000)<1.9$, respectively. Redder galaxies generally show a stronger redshift evolution of $\lambda_{\text {Edd }}$ distribution than bluer ones.

Recently, there have been a number of studies revealing that the emergence of GV galaxies lying between the quiescent RS and the strong star-forming BC populations may be closely related to AGN activities. For example, local AGNs or changing-look quasars are usually preferentially found in GV galaxies (e.g., Lacerda et al. 2020; Liu et al. 2021; Zhang et al. 2021; Dodd et al. 2021). The other possible connection between GV galaxies and AGNs is that both of the two populations may be experiencing "morphological compaction" (e.g., Barro et al. 2013, 2015; Kocevski et al. 2017; Lu et al. 2021). But till now, there has been little direct evidence about AGN activities inevitably suppressing the global galaxy star formation, especially at high redshifts (e.g., Torres-Papaqui et al. 2020). There are indeed some studies about AGN-driven star-formation quenching in the local universe. For example, based on the Calar Alto Legacy Integral Field spectroscopy Area survey (CALIFA) integral-field unit (IFU) data, Kalinova et al. (2021) traced the ionised hydrogen distribution maps in kiloparsec (kpc) scales to study galaxy quenching mechanisms. Their results as well as some relevant studies (e.g., Appleby et al. 2020; Brownson et al. 2020; Zewdie et al. 2020) reinforced the "inside-out" quenching scenario where quenching begins from galaxy centers. However, this evidence is still somewhat indirect, since when they examined the properties of those galaxies with weak and strong AGN emission features during their quenching processes, they do not find any observable differences. The result of AGN lifetime estimation in galaxies of different extinction-corrected rest-frame $U V$ colors in this paper hence may 
complement the recent discoveries of AGN and host-galaxy coevolution, which provides us a clue to gain more information about AGN feedback at high redshifts.

To examine whether AGN accretion activities drive quenching in star-forming galaxies, we compare the AGN lifetime distribution in a certain population of galaxies with the respective typical galaxy color transitional timescale (Lin et al. 2021). In Lin et al. (2021), we adopted the same selection criteria as Wang et al. (2017) to divide all COSMOS/UltraVISTA galaxies into populations of different extinctioncorrected rest-frame $U V$ colors. Then, we estimated the lifetime (i.e., color transitional timescale) of each galaxy population based on its derived duty cycle. To achieve this, we first assumed that for each dark matter halo that has a similar mass with the measured host halo $\left(M_{h}\right)$ of one of the galaxy populations in Lin et al. (2019), the galaxy residing in it would undergo the phase (color) of the respective galaxy population. After that, we counted the total number of the halos that have similar $M_{h}$ to the host halo of that galaxy population in Lin et al. (2019), and computed the fraction of halos hosting those galaxies, i.e., the duty cycle. The resulting lifetimes (i.e., color transitional timescales of the three galaxy populations) were shown to be well consistent with the results computed through SED modeling (for more details, we refer the readers to Lin et al. 2021).

We show the typical galaxy color transitional timescales at each redshift as the horizontal color-filled areas in each panel of Figure 3. It is clear that for GV galaxies, the region where the AGN lifetime and galaxy color transitional timescale overlap lies at low $\lambda_{\text {Edd }}$ at lower redshifts, but moves to high $\lambda_{\mathrm{Edd}}$ at higher redshifts $(z \sim 2)$. In other words, the typical color transitional timescales of GV galaxies coincide with the lifetimes of AGNs of different accretion activities at different redshifts. At higher redshifts, the timescale that strong AGN accretion persists is almost identical with the color transitional timescale of GV galaxies, which implies that strong AGN feedback (e.g., quasar/radiation-mode feedback, Sijacki et al. 2008; Fabian 2012; King \& Pounds 2015; Cielo et al. 2018) is very likely to be accompanied with the color transition in GV galaxies. At lower redshifts, the quenching timescale of GV galaxies becomes longer (e.g., Schawinski et al. 2014; Smethurst et al. 2015; Belfiore et al. 2017; Rowlands et al. 2018; Trussler et al. 2020). Given that the lifetimes of all AGNs are anti-correlated with the accretion rates, if the quenching (or galaxy color transition) here is still driven by AGN accretion activities, then weakly accreting AGNs should play the dominant role (e.g., Shangguan et al. 2018). This result can be reflected in Figure 2, where the slope of Eddington ratio distribution of GV galaxies becomes steeper at lower redshifts.

In comparison with $\mathrm{GV}$ galaxies, the typical color transitional timescale of $\mathrm{BC}$ galaxies always overlaps with their AGN lifetime distribution on the low $\lambda_{\text {Edd }}$ side at whatever redshift. We reckon that AGNs with high accretion rates would not have a quite significant effect on the color transition of the BC galaxy population. This is because, although strong AGN feedback activities are likely to influence the star formation of the whole star-forming galaxy population, these extremely accreting AGNs only make up a small fraction of the total amount of AGNs within BC galaxies. Besides, BC galaxies are usually discovered to be gas rich (Stark et al. 1986; Taylor et al. 1999; Boselli et al. 2014; Cañameras et al. 2018; Tacconi et al. 2018), which may effectively slow down the feedback wind driven by central AGNs.

We need to clarify that, although the inflowing gas within BC galaxies will feed the central SMBHs at a longer time than GV galaxies, which may further trigger subsequential strong AGN feedback, it does not mean that all the AGNs among BC galaxies are strongly accreting AGNs at the time we observed them. Hickox et al. (2009) proposed that the behaviors of AGNs and their host galaxies are accompanied by the growth of their host dark matter halos. This scenario has been supported by a number of studies, (e.g., Behroozi et al. 2013; Cai et al. 2013; Moster et al. 2013; Vogelsberger et al. 2014; Chen et al. 2020). According to this scenario, the strong accretion and feedback activity of an AGN can only be triggered when the mass of its host halo reaches a critical value of $10^{12} \sim 10^{13} M_{\odot}$ (see Figure 16 in Hickox et al. 2009). For most BC galaxies, their typical halo masses have just reached the lower limit of this value (Lin et al. 2019). They also have relatively smaller stellar masses (Lin et al. 2019), and thus smaller black hole masses compared with GV galaxies (if we assume a uniform black hole mass to stellar mass ratio). Thus it is more likely that these less massive black holes among BC galaxies are accreting in a smooth and peaceful way. They spend their long lifetimes in a relatively weaker accretion 


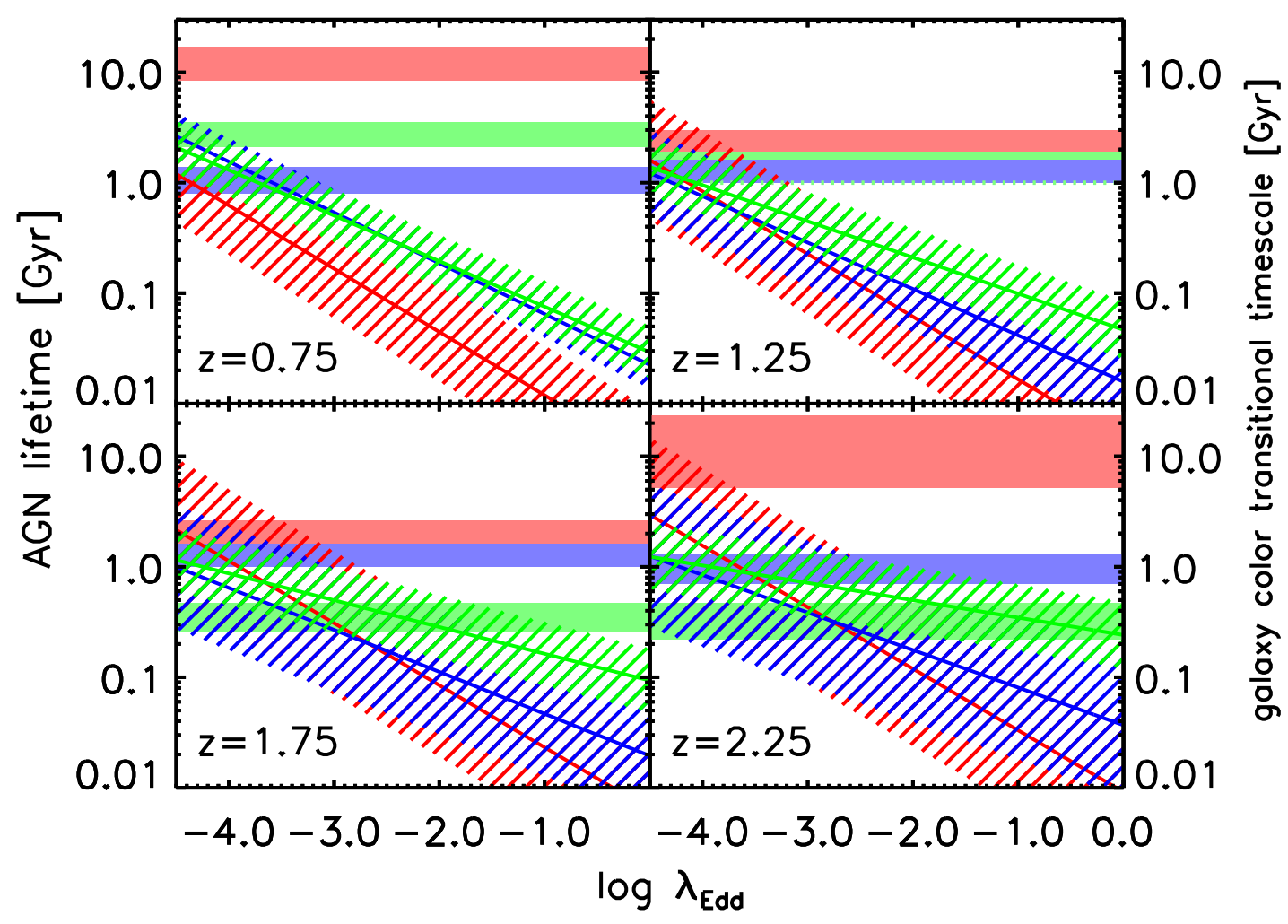

Fig. 3: The lifetimes of AGNs among galaxies of different extinction-corrected rest-frame $U V$ colors at $z=0.75, z=1.25, z=1.75$, and $z=2.25$ in comparison with the typical color transitional timescales of these galaxies. Solid colored lines with the line-filled regions show the lifetimes and the corresponding uncertainties of AGNs among the RS, GV and BC galaxies derived from their Eddington ratio distributions following Equation 1 and Equation 3. The horizontal color-filled regions show the typical color transitional timescales of the three galaxy populations at the corresponding redshifts taken from Lin et al. (2021).

mode, as proposed in some studies (e.g., Lapi et al. 2014) that strong near Eddington accretion cannot persist long, and most of such accretions are more likely to happen in more massive SMBH systems. Therefore, although some strongly accreting AGNs may act on the star formation in some specific BC galaxies, they are not able to cause the entire $\mathrm{BC}$ galaxy population to transform its color in a short time. For the population of RS galaxies, we consider them as a stable phase according to the quenching scenario, so that their color transitional timescales are theoretically infinite, which can be much longer than the estimates shown in Figure 3.

An alternative demonstration is to compare the lifetimes of AGNs with the galaxy color transitional timescales as a function of redshift, which is shown in Figure 4. Filled circles with error bars show the typical color transitional timescales of the three galaxy populations at $z \sim 0.8,1.2,1.7$, and 2.2. Thick solid curves show the best-fit color transitional lifetime parameters to reproduce the exact photometricredshift distributions of the three galaxy populations, based on a pre-assumed halo mass function (Tinker et al. 2008) and a median halo mass growth rate in the Millennium-II simulations (Fakhouri et al. 2010). The typical lifetimes of AGNs with the accretion rates fixed to $\lambda_{\text {Edd }} \geq 1,0.1,0.01,0.001,0.0001$ for each galaxy population are shown as the dash-dotted lines of each color in the same panel. These lifetimes are derived in a similar way as computing the AGN lifetimes as a function of accretion rate (see Figure 3). The only difference is that here, we interpolate the AGN lifetime as a function of redshift 


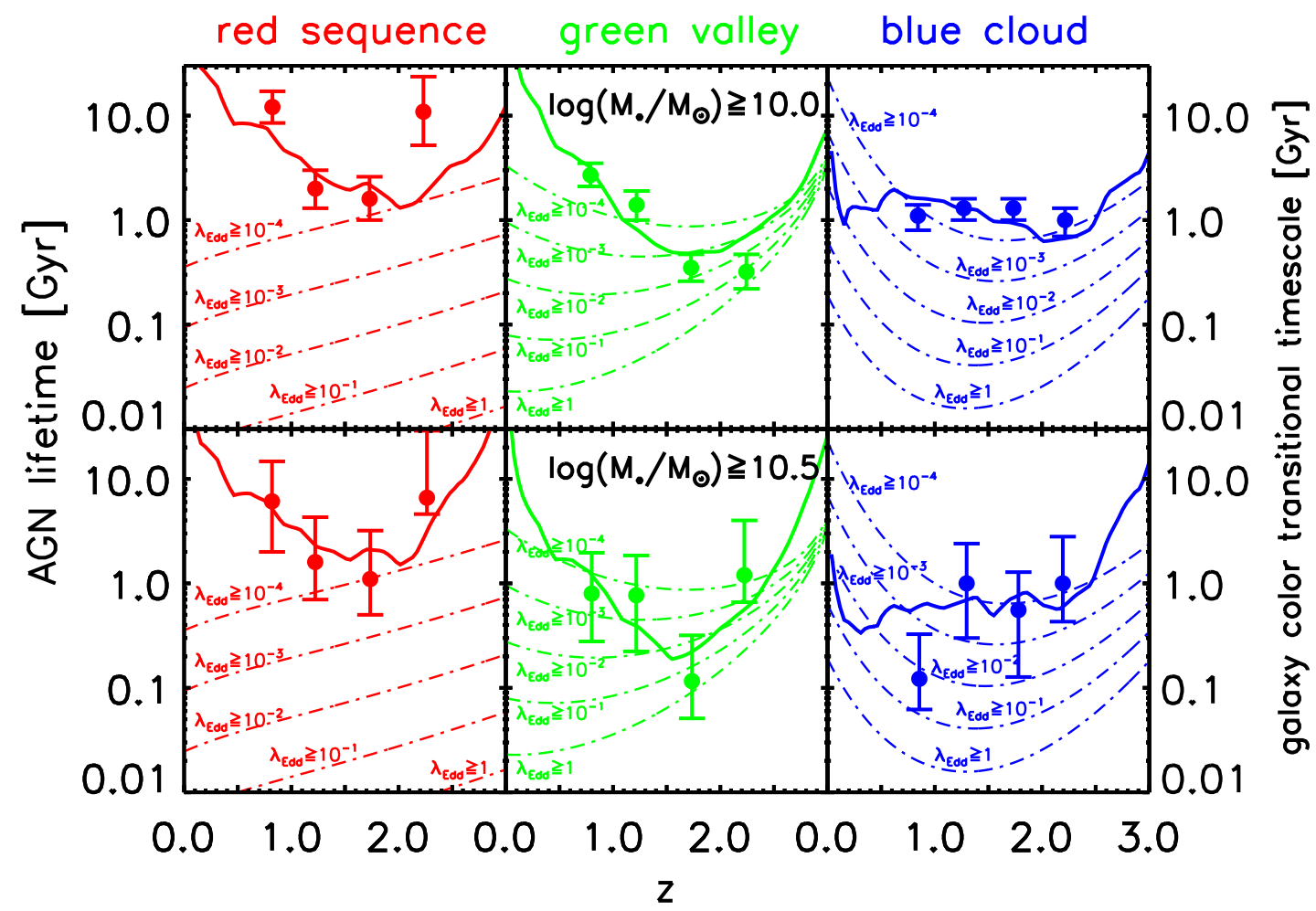

Fig. 4: The comparison between the lifetimes of AGNs and the typical color transitional timescales of RS, BC and GV galaxies as a function of redshift. Upper panels show the results of host galaxies selected with $\log \left(M_{*} / M_{\odot}\right) \geq 10.0$, while lower panels show the results of host galaxies selected with $\log \left(M_{*} / M_{\odot}\right) \geq 10.5$. Filled circles with error bars in each panel show the lifetimes (typical color transitional timescales) of galaxies of different colors (Lin et al. 2021). Thick solid curves show the bestfit lifetime parameters as a function of redshift to reproduce the exact photometric-redshift distributions of the three galaxy populations, based on a pre-assumed halo mass function (Tinker et al. 2008), and the median halo mass growth rate from the Millennium-II simulation (see Lin et al. 2021 for more details). The thin dash-dotted lines in each panel show the lifetimes of the AGNs in the three galaxy populations, with the accretion rates fixed to $\lambda_{\mathrm{Edd}} \geq 1,10^{-1}, 10^{-2}, 10^{-3}, 10^{-4}$. The lifetimes of the AGNs with high $\lambda_{\text {Edd }}$ overlap with the typical color transitional timescales of GV galaxies at high redshifts $(z \sim 1.5-2)$. While among BC galaxies, the time when the lifetime curves of the high $\lambda_{\mathrm{Edd}}$ AGNs reach the typical color transitional timescales of $\mathrm{BC}$ galaxies seems to come much later, i.e., at $z<1$. It means that strong AGN feedback is very likely to be accompanied with the color transition in GV galaxies at high redshifts, reinforcing the coevolution scenario between central AGNs and host galaxies. In comparison, among $\mathrm{BC}$ galaxies, the significant star-formation regulation due to the strong central AGN accretion activities seems to be put off.

rather than accretion rate. During each interpolation, we allow redshift to vary and keep the accretion rate limit $\lambda_{\text {Edd }}$ fixed.

A notable feature in Figure 4 is that the lifetimes of AGNs in galaxies of different colors exhibit distinct redshift evolution behaviors. Among RS galaxies, for any given thresholds of their accretion rates, the lifetimes of AGNs increase smoothly with redshift. The situations are different in GV and BC galaxies. Among GV galaxies, while the lifetimes of the strongestly accreting AGNs still increase monotonically with redshift, those with medium and low accretion rates appear to have minimum life- 
times at $z \sim 1-2$. This trend is even more significant among BC galaxies, where the AGN lifetimes of all accretion rates reach the minimums at $z \sim 1-2$. These results indicate that observable strong and persistent AGN accretion activities are most likely to be detected among the high-redshift $(z \sim 2) \mathrm{GV}$ galaxies and some of the lower-redshift BC galaxies.

Further comparison between the AGN lifetimes and the typical color transitional timescales of galaxies at each redshift reveals that the lifetimes of the strongly accreting AGNs among GV galaxies reach the typical color transitional timescales of GV galaxies at $z \sim 1.5-2$, which is considered as the peak of the global black hole accretion activities and the peak of the global star-formation activities across cosmic time (Delvecchio et al. 2014; Heckman \& Best 2014; Madau \& Dickinson 2014; Ma \& Yan 2015; Sijacki et al. 2015; Zakamska et al. 2016). The result is consistent with the scenario where strongly accreting AGNs act on governing the gas depletion in GV galaxies at high redshifts, transforming the galaxy colors, making SMBHs and galaxies coevolve with each other during the cosmic time. As a comparison, in BC galaxies, the time that strong AGN accretions significantly act on galaxy color transition comes much later $(z<1)$. The trend is clearer in more massive BC galaxies, i.e., $\log \left(M_{*} / M_{\odot}\right) \geq 10.5$, which is shown in the lower-right panel of Figure 4 . This result agrees well with the studies of Gonçalves et al. (2012) and Fritz et al. (2014), who found a rapid acceleration of quenching among BC galaxies at $z<1$, through analyzing the evolution of their luminosity/stellar mass functions.

Our study of AGN lifetimes is based on their Eddington ratio distributions. As mentioned in Section 2, the condition of $t \ll t_{H}$ in Equation 1 may not be established in the early universe, which would probably result in the under-prediction of the true lifetimes of the AGNs at high redshifts. Nevertheless, this effect would not alter the main results and conclusions of this paper, since it only causes the lifetime of AGNs to rise at the high-redshift end in Figure 4, which makes the lifetimes of the strongestly accreting AGNs better match the typical color transitional timescales of the GV galaxies at these redshifts.

At lower redshifts, however, it is also possible that the feedback activities of the central black holes may not be the only and main cause of galaxy quenching, since AGNs among galaxy populations of all colors spend their long lifetimes on the weakly accreting phase (see Figure 3). Various hydrodynamical simulations considering AGN-driven quenching also tend to over-produce the quiescent GV galaxies (i.e., GV galaxies with lower specific star-formation rates) compared with the observations (Angthopo et al. 2021), which suggests that there may be some additional mechanisms that dominate quenching at low redshifts, e.g., environmental quenching (Schawinski et al. 2014; Belfiore et al. 2017; Kacprzak et al. 2021), halo quenching (Bluck et al. 2020), or a modified more complex AGN-driven quenching mechanism such as halo mass threshold and black hole seed mass triggering AGN feedback (Davé et al. 2020; Trussler et al. 2020; Angthopo et al. 2021).

\section{SUMMARY AND CONCLUSIONS}

In this paper, we follow the script proposed by Hopkins \& Hernquist (2009) to study the lifetimes of AGNs in the galaxies of different extinction-corrected rest-frame $U V$ colors, based on their derived Eddington ratio distributions in Wang et al. (2017). First, we compare the Eddington ratio distribution of the entire galaxy population in Wang et al. (2017) with the semi-analytic and observational results in Hopkins \& Hernquist (2009) and Kauffmann \& Heckman (2009) at lower redshifts, (i.e., $z=1$ and $z=0)$. We find that they are well consistent with each other.

Second, we make a systematic comparison between the Eddington ratio distributions of the AGNs among the galaxies of different $U V$ colors. We also compare them to the $\lambda_{\text {Edd }}$ distributions of the AGNs among local galaxies of several respective $D_{n}(4000)$ indexes (Kauffmann \& Heckman 2009) to study the evolution of $\lambda_{\mathrm{Edd}}$ as a function of redshift. The $\lambda_{\mathrm{Edd}}$ distribution of the AGNs among RS galaxies exhibits the strongest evolution among the three galaxy populations; while for BC galaxies, we do not detect any significant evolution of the $\lambda_{\mathrm{Edd}}$ distribution with cosmic time, indicating that there may be no apparent difference in the black hole accretion triggering mechanisms among these galaxies. 
Third, we study the lifetimes of the X-ray AGNs among the galaxies of different extinctioncorrected rest-frame $U V$ colors. The typical lifetimes of X-ray AGNs among these galaxies are generally from several tens of million years to $\sim 1 \mathrm{Gyr}$, with strongerly accreting sources having shorter lifetimes. On the low accretion rate side, there are no observable differences in the estimated lifetimes among the galaxies of all colors. However, on the high accretion rate side, it is clear that the AGNs among GV galaxies have the longest lifetimes compared to those of RS and BC galaxies, suggesting that strongly accreting AGNs are rather common in GV galaxies, especially at higher redshifts $(z \sim 2)$.

Fourth, we compare the lifetimes of the AGNs with the typical color transitional timescales of the galaxies of all colors derived in Lin et al. (2021) as a function of $\lambda_{\text {Edd }}$. The typical color transitional timescales of GV galaxies are consistent with the lifetimes of the weakly and strongly accreting AGNs at lower and higher redshifts, respectively, indicating an enhancement of AGN feedback activities among these galaxies at higher redshifts. However, for $\mathrm{BC}$ galaxies, their typical color transitional timescales always overlap with the lifetimes of lower accretion rate AGNs, which means that strong AGN feedback activities among these galaxies, accounting for a small fraction, are not able to change their global in-situ colors within a short period of time.

Finally, we study the AGN lifetimes and compare them to the typical color transitional timescales of galaxies as a function of redshift. The lifetimes of the AGNs among the galaxies of different colors exhibit distinct redshift evolution behaviors. It seems clear that strong and persistent AGN accretion is most detectable among those high-redshift $(z \sim 2) \mathrm{GV}$ galaxies and some of the lower-redshift $(z<1)$ $\mathrm{BC}$ galaxies. The lifetimes of the strongly accreting AGNs among GV galaxies are almost identical with the typical color transitional timescales of their hosts at $z \sim 1.5-2$. This redshift coincides with the peaks of global black hole accretion activities and galaxy star-formation activities during the entire cosmic time. While in BC galaxies, strong AGN accretion activities are likely to have observable effects on the color transition of their hosts at lower redshifts $(z<1)$. Our results support the quenching scenario in massive galaxies where strong AGN accretion activities help to remove the gas contents in some intermediate-redshift star-forming galaxies, making SMBHs and their hosts coevolve with each other.

Acknowledgements We thank the referee for helpful comments. X.Z.L., Y.Q.X., and H.A.N.L. acknowledge support from NSFC-12025303, 11890693, 11421303, 12003031, the CAS Frontier Science Key Research Program (QYZDJ-SSW-SLH006), the K.C. Wong Education Foundation, and the science research grants from the China Manned Space Project with NO. CMS-CSST-2021-A06. H.A.N.L. acknowledges support from the Chinese Academy of Sciences President's International Fellowship Initiative (Grant No. 2019PM0020).

\section{References}

Adelberger, K. L., Steidel, C. C., Pettini, M., et al. 2005, ApJ, 619, 6972

Aird, J., Coil, A. L., Moustakas, J., et al. 2012, ApJ, 746, 905

Alexander, D. M., Bauer, F. E., Brandt, W. N., et al. 2003, AJ, 126, 5395

Angthopo, J., Negri, A., Ferreras, I., et al. 2021, MNRAS, 502, 368512

Appleby, S., Davé, R., Kraljic, K., Anglés-Alcázar, D., \& Narayanan, D. 2020, MNRAS, 494, 60538

Barone, L. T., Heithausen, A., Hüttemeister, S., Fritz, T., \& Klein, U. 2000, MNRAS, 317, 6497

Barro, G., Faber, S. M., Pérez-González, P. G., et al. 2013, ApJ, 765, 1048

Barro, G., Faber, S. M., Koo, D. C., et al. 2015, arXiv:1509.00469 8

Behroozi, P. S., Wechsler, R. H., \& Conroy, C. 2013, ApJ, 770, 579

Belfiore, F., Maiolino, R., Maraston, C., et al. 2017, MNRAS, 466, 2570 9, 12

Bell, E. F., Wolf, C., Meisenheimer, K., et al. 2004, ApJ, 608, 7522

Bell, M. B. 2002, ApJ, 567, 8012

Blanton, M. R., Hogg, D. W., Bahcall, N. A., et al. 2003, ApJ, 594, 1862

Bluck, A. F. L., Maiolino, R., Piotrowska, J. M., et al. 2020, MNRAS, 499, 23012

Borch, A., Meisenheimer, K., Bell, E. F., et al. 2006, A\&A, 453, 8692 
Boselli, A., Cortese, L., Boquien, M., et al. 2014, A\&A, 564, A67 7, 9

Brammer, G. B., Whitaker, K. E., van Dokkum, P. G., et al. 2009, ApJ, 706, L173 2

Brownson, S., Belfiore, F., Maiolino, R., Lin, L., \& Carniani, S. 2020, MNRAS, 498, L66 8

Cai, Z.-Y., Lapi, A., Xia, J.-Q., et al. 2013, ApJ, 768, 219

Cañameras, R., Yang, C., Nesvadba, N. P. H., et al. 2018, A\&A, 620, A61 7, 9

Cassata, P., Cimatti, A., Kurk, J., et al. 2008, A\&A, 483, L39 2

Chang, Y.-Y., van der Wel, A., da Cunha, E., \& Rix, H.-W. 2015, ApJS, 219, 82

Chen, Z., Fang, G., Lin, Z., et al. 2020, arXiv e-prints, arXiv:2011.05528 9

Cielo, S., Bieri, R., Volonteri, M., Wagner, A. Y., \& Dubois, Y. 2018, MNRAS, 477, 13369

Cirasuolo, M., McLure, R. J., Dunlop, J. S., et al. 2007, MNRAS, 380, 5852

Cresci, G., Mainieri, V., Brusa, M., et al. 2015, ApJ, 799, 822

Croom, S. M., Richards, G. T., Shanks, T., et al. 2009, MNRAS, 399, 17552

Davé, R., Crain, R. A., Stevens, A. R. H., et al. 2020, MNRAS, 497, 14612

Delvecchio, I., Gruppioni, C., Pozzi, F., et al. 2014, MNRAS, 439, 273612

Dodd, S. A., Law-Smith, J. A. P., Auchettl, K., Ramirez-Ruiz, E., \& Foley, R. J. 2021, ApJ, 907 , L21 8

Fabian, A. C. 2012, ARA\&A, 50, 4559

Fakhouri, O., Ma, C.-P., \& Boylan-Kolchin, M. 2010, MNRAS, 406, 226710

Fang, J. J., Faber, S. M., Koo, D. C., \& Dekel, A. 2013, ApJ, 776, 632

Ferrarese, L., \& Merritt, D. 2000, ApJ, 539, L9 1

Franzetti, P., Scodeggio, M., Garilli, B., et al. 2007, A\&A, 465, 7112

Fritz, A., Scodeggio, M., Ilbert, O., et al. 2014, A\&A, 563, A92 12

Gebhardt, K., Bender, R., Bower, G., et al. 2000, ApJ, 539, L13 1

Giallongo, E., Salimbeni, S., Menci, N., et al. 2005, ApJ, 622, 1162

Gonçalves, T. S., Martin, D. C., Menéndez-Delmestre, K., Wyder, T. K., \& Koekemoer, A. 2012, ApJ, 759, 6712

Graham, A. W. 2012, ApJ, 746, 1132

Heckman, T. M., \& Best, P. N. 2014, ARA\&A, 52, 58912

Hickox, R. C., Jones, C., Forman, W. R., et al. 2009, ApJ, 696, 891 2, 9

Hopkins, P. F., \& Hernquist, L. 2009, ApJ, 698, 1550 2, 3, 5, 6, 12

Hopkins, P. F., Hernquist, L., Cox, T. J., et al. 2005, ApJ, 630, 705 2, 4

Hopkins, P. F., Hernquist, L., Cox, T. J., et al. 2006a, ApJS, 163, 1 4, 5

Hopkins, P. F., Hernquist, L., Cox, T. J., et al. 2006b, ApJ, 639, 7004

Hopkins, P. F., Richards, G. T., \& Hernquist, L. 2007, ApJ, 654, 731 2, 5

Ishibashi, W., \& Fabian, A. C. 2012, MNRAS, 427, 29982

Jones, M. L., Hickox, R. C., Black, C. S., et al. 2016, ApJ, 826, 125

Kacprzak, G. G., Nielsen, N. M., Nateghi, H., et al. 2021, MNRAS, 500, 228912

Kalinova, V., Colombo, D., Sánchez, S. F., et al. 2021, A\&A, 648, A64 8

Kauffmann, G., \& Heckman, T. M. 2009, MNRAS, 397, 135 5, 6, 7, 8, 12

King, A., \& Pounds, K. 2015, ARA\&A, 53, 1159

Kocevski, D. D., Barro, G., Faber, S. M., et al. 2017, ApJ, 846, 1128

Kormendy, J., Bender, R., \& Cornell, M. E. 2011, Nature, 469, 3741

Kormendy, J., \& Ho, L. C. 2013, ARA\&A, 51, 511 1, 2

Lacerda, E. A. D., Sánchez, S. F., Cid Fernandes, R., et al. 2020, MNRAS, 492, 30738

Lapi, A., Raimundo, S., Aversa, R., et al. 2014, ApJ, 782, 6910

Le, H. A. N., Pak, S., Kaplan, K., et al. 2017a, ApJ, 841, 132

Le, H. A. N., Woo, J.-H., Son, D., et al. 2017b, ApJ, 851, 82

Leauthaud, A., Tinker, J., Bundy, K., et al. 2012, ApJ, 744, 1592

Lee, G.-H., Hwang, H. S., Lee, M. G., et al. 2015, ApJ, 800, 802

Lin, X., Fang, G., Cai, Z.-Y., et al. 2019, ApJ, 875, 83 2, 4, 9

Lin, X., Fang, G., Xue, Y., Fan, L., \& Kong, X. 2021, ApJ, 911, 59 2, 4, 5, 9, 10, 11, 13

Liu, W.-J., Lira, P., Yao, S., et al. 2021, ApJ, 915, 638

Lu, S., Fang, G., Gu, Y., et al. 2021, ApJ, 913, 818 
Ma, Z., \& Yan, H. 2015, ApJ, 811, 5812

Madau, P., \& Dickinson, M. 2014, ARA\&A, 52, 41512

Martig, M., Bournaud, F., Teyssier, R., \& Dekel, A. 2009, ApJ, 707, 2502

Martini, P. 2004, Coevolution of Black Holes and Galaxies, 1692

Merloni, A., Bongiorno, A., Bolzonella, M., et al. 2010, ApJ, 708, 1372

Miyaji, T., Hasinger, G., \& Schmidt, M. 2000, A\&A, 353, 252

Moster, B. P., Naab, T., \& White, S. D. M. 2013, MNRAS, 428, 31219

Perets, H. B. 2009, ApJ, 698, 13307

Portegies Zwart, S. F. 2000, ApJ, 544, 4377

Reipurth, B., Bally, J., \& Devine, D. 1997, AJ, 114, 27087

Rowlands, K., Wild, V., Bourne, N., et al. 2018, MNRAS, 473, 1168 2, 9

Sage, L. J., Salzer, J. J., Loose, H. H., \& Henkel, C. 1992, A\&A, 265, 197

Saglia, R. P., Opitsch, M., Erwin, P., et al. 2016, ApJ, 818, 47 1, 2

Schawinski, K. Urry, C. M., Virani, S., Coppi, P., et al. 2010, ApJ, 284, 7112

Schawinski, K., Urry, C. M., Simmons, B. D., et al. 2014, MNRAS, 440, 889 2, 9, 12

Shangguan, J., Ho, L. C., \& Xie, Y. 2018, ApJ, 854, 1589

Shin, J., Woo, J.-H., Chung, A., et al. 2019, ApJ, 881, 1472

Sijacki, D., Pfrommer, C., Springel, V., \& Enßlin, T. A. 2008, MNRAS, 387, 14039

Sijacki, D., Vogelsberger, M., Genel, S., et al. 2015, MNRAS, 452, 57512

Silk, J., \& Rees, M. J. 1998, A\&A, 331, L1 2

Silverman, J. D., Mainieri, V., Lehmer, B. D., et al. 2008, ApJ, 675, 10252

Smethurst, R. J., Lintott, C. J., Simmons, B. D., et al. 2015, MNRAS, 450, 4359

Stark, A. A., Knapp, G. R., Bally, J., et al. 1986, ApJ, 310, 660 7, 9

Strateva, I., Ivezić, Ž., Knapp, G. R., et al. 2001, AJ, 122, 18612

Sun, M., Trump, J. R., Brandt, W. N., et al. 2015, ApJ, 802, 142

Tacconi, L. J., Genzel, R., Saintonge, A., et al. 2018, ApJ, 853, 179 7, 9

Taylor, C. L., Hüttemeister, S., Klein, U., \& Greve, A. 1999, A\&A, 349, 424 7, 9

Tinker, J., Kravtsov, A. V., Klypin, A., et al. 2008, ApJ, 688, 709 10, 11

Torres-Papaqui, J. P., Coziol, R., Romero-Cruz, F. J., et al. 2020, AJ, 160, 176 2, 8

Trump, J. R., Sun, M., Zeimann, G. R., et al. 2015, ApJ, 811, 265

Trussler, J., Maiolino, R., Maraston, C., et al. 2020, MNRAS, 491, 5406 9, 12

Vogelsberger, M., Genel, S., Springel, V., et al. 2014, MNRAS, 444, 15189

Wang, T., Elbaz, D., Alexander, D. M., et al. 2017, A\&A, 601, A63 2, 3, 5, 6, 7, 9, 12

Wetzel, A. R., Tinker, J. L., \& Conroy, C. 2012, MNRAS, 424, 2322

Whitaker, K. E., Labbé, I., van Dokkum, P. G., et al. 2011, ApJ, 735, 862

Xue, Y. Q., Brandt, W. N., Luo, B., et al. 2010, ApJ, 720, 3682

Xue, Y. Q., Luo, B., Brandt, W. N., et al. 2011, ApJS, 195, 105

Young, J. S., Xie, S., Kenney, J. D. P., \& Rice, W. L. 1989, ApJS, 70, 6997

Yu, Q., \& Lu, Y. 2004, ApJ, 602, 6034

Zakamska, N. L., Hamann, F., Pâris, I., et al. 2016, MNRAS, 459, 314412

Zewdie, D., Pović, M., Aravena, M., Assef, R. J., \& Gaulle, A. 2020, MNRAS, 498, 43458

Zhang, Z., Wang, H., Luo, W., et al. 2021, A\&A, 650, A155 8

Zolotov, A., Dekel, A., Mandelker, N., et al. 2015, MNRAS, 450, 23272

Zubovas, K., Nayakshin, S., King, A., \& Wilkinson, M. 2013, MNRAS, 433, 30792 